\title{
Outcomes of Advanced Retinoblastoma Treated with Local Salvage Treatment; a Retrospective Case Series
}

\author{
Palwasha Rehman, Saadiya Javed Khan \\ Department of Pediatric Oncology, Shaukat Khanum Cancer Hospital and Research Centre, Lahore, \\ Pakistan
}

Received: 27 September 2019/Accepted: 16 December 2019

\section{G openaceess \\ Correspondence: Palwasha} Rehman, Department of Pediatric Oncology, Shaukat Khanum Cancer Hospital and Research Centre, Lahore, Pakistan. Email: Palwasharehman@skm. org.pk

Citation: Rehman P, Khan SJ. Outcomes of advanced retinoblastoma treated with local salvage treatment; a retrospective case series. J Cancer Allied Spec [Internet]. 2020 Jan.6; 6(1):e1002957. https://doi.org/10.37029/jcas. v6i1.283

Copyright: (c) 2020 Rehman and Khan. This is an open access article distributed under the terms of the Creative Commons Attribution License, which permits unrestricted use, distribution, and reproduction in any medium, provided the original author and source are credited.

Funding: This research received no specific grant from any funding agency in the public, commercial or not-for-profit sectors.

Competing interests: Nill.

\section{Introduction}

Retinoblastoma (RB) is the most common intraocular tumour of childhood. ${ }^{[1]}$ No variation is seen among the age and gender at presentation. An estimated 5000 new cases are reported every year. A relatively recent population-based cancer survey has reported that the age-adjusted incidence of $\mathrm{RB}$ in India alone is 1.3-12.3/million children. ${ }^{[2]} \mathrm{RB}$

\begin{abstract}
Introduction: Retinoblastoma (RB) is the most common intraocular malignancy arising from the developing retina and occurs in approximately one of every 15,000-20,000 births. With the introduction of the intraarterial chemotherapy (IAC), the 5-year overall survival of children with $\mathrm{RB}$ is $99 \%$, though in low- and middle-income countries, it rarely exceeds $35 \%$ due to limited resources and lack of expertise. The aim of this study was to determine the outcome of local salvage in advanced RB. Materials and Methods: A retrospective analysis was conducted on children diagnosed with advanced RB that had local salvage therapy along with systemic chemotherapy from January 2015 to January 2018 at Shaukat Khanum Memorial Cancer Hospital and Research Centre, Lahore, Pakistan. Results: Fifteen patients were included in the study, among these 10 were male. The median age of presentation was 20 months (range 2-40 months). Among participants, 11 patients had bilateral RB. Fourteen patients received local control along with systemic chemotherapy. Relapse disease was seen in 12 patients and 2-year disease-free survival (DFS) was 20\%. Conclusion: The results of the present study suggest that centres lacking the resources for IAC should treat advance cases of RB with an upfront or early enucleation.
\end{abstract}

Key words: Eye, neoplasm, paediatrics, retinoblastoma, treatment is malignancy associated with germline or somatic mutation. Germline mutations can present as early as 1 -month age. However, sporadic mutations are detected at approximately 24 months of age..$^{[3]}$ Leucocoria (white reflex) is the most common presenting sign, which is noticed when the tumour is still contained within the eye. When detected at an early age, prompt intervention can save the eye. However, delayed diagnosis can lead to invasion 
of the optic nerve and widespread metastatic disease. ${ }^{[4]}$

There have been many advances in the management of RB. These include systemic chemotherapy, external beam radiotherapy and intravitreal chemotherapy. At present, Intraophthalmic artery chemotherapy (OAC) is considered as the first line of therapy for the advance and recurrent RB..$^{[5]}$ However, in the absence of availability of OAC, enucleation remains the standard for the advanced disease.

The aim of this retrospective analysis was to determine the outcome of salvage treatment in advanced RB in a setting where OAC was not available.

\section{Materials and Methods}

A retrospective chart review was performed of patients who underwent local salvage therapy for the management of advanced RB Shaukat Khanum Memorial Cancer Hospital and Research Centre, Lahore, Pakistan, between January 2015 and January 2018. Approval from the local Institutional Review Board was obtained (EX-12-09-19-01).

All patients had undergone a comprehensive clinical assessment comprising a detailed history, thorough examination and clinicoradiopathological evaluation. This comprised magnetic resonance imaging (MRI) of the brain, evaluation under anaesthesia (EUA), bilateral bone marrow biopsy and cerebrospinal fluid analysis. Local staging of the disease was performed, based on the International Classification of Retinoblastoma (IRCB) [Table 1]. ${ }^{[6]}$ Group D and Group E diseases were labelled as locally advanced disease.

Patients that were diagnosed with advance RB were started on systemic chemotherapy adapted from the guidelines of the United Kingdom Children's Cancer

Table 1: International Classification of Retinoblastoma. ${ }^{[6]}$

\begin{tabular}{|c|c|}
\hline \multicolumn{2}{|c|}{ International Classification of Retinoblastoma } \\
\hline Group A & $\begin{array}{l}\text { Small intraretinal tumour away from the fovea and disc } \\
\text { All tumours are } 3 \mathrm{~mm} \text { or smaller in greatest dimension, confined to the retina and } \\
\text { All tumours are located further than } 3 \mathrm{~mm} \text { from the foveola and } 1.5 \mathrm{~cm} \text { from the optic disc }\end{array}$ \\
\hline Group B & $\begin{array}{l}\text { All remaining discrete tumours confined to the retina } \\
\text { All other tumours confined to the retina not in Group A } \\
\text { Tumour-associated subretinal fluid }<3 \mathrm{~mm} \text { from the tumour with no subretinal seeding. }\end{array}$ \\
\hline Group C & $\begin{array}{l}\text { Discrete local disease with minimal subretinal or vitreous seeding } \\
\text { Tumour(s) are discrete } \\
\text { Subretinal fluid, present or past without seeding up to } 1 / 4 \text { retina } \\
\text { Local fine vitreous seeding may be present close to discrete tumour. } \\
\text { Local subretinal seeding }<3 \mathrm{~mm} \text { (2DD) from the tumour }\end{array}$ \\
\hline Group D & $\begin{array}{l}\text { Diffuse disease with significant vitreous and subretinal seeding } \\
\text { Tumour(s) may be massive or diffuse } \\
\text { Subretinal fluid, present or past without seeding, involving up to total retinal detachment } \\
\text { Diffuse or massive vitreous disease may include 'greasy' seeds or avascular tumour masses. } \\
\text { Diffuse subretinal seeding may include subretinal plaques or tumour nodules. }\end{array}$ \\
\hline Group E & $\begin{array}{l}\text { Presence of any one or more of the following poor prognosis features } \\
\text { Tumour touching the iris } \\
\text { Tumour anterior to anterior vitreous face involving ciliary body or anterior segment } \\
\text { Diffuse infiltrating retinoblastoma } \\
\text { Neovascular glaucoma } \\
\text { Opaque media from haemorrhage } \\
\text { Tumour necrosis with aseptic orbital cellulitis } \\
\text { Phthisis bulbi }\end{array}$ \\
\hline
\end{tabular}


Study Group - RB working Group. ${ }^{[7]}$ The initial intent of the treatment was globe preservation. All patients were started on JOE chemotherapy. This consists of vincristine $\left(1.5 \mathrm{mg} / \mathrm{m}^{2}\right)$, carboplatin $\left(600 \mathrm{mg} / \mathrm{m}^{2}\right)$ and etoposide $\left(300 \mathrm{mg} / \mathrm{m}^{2}\right)$. Patients received from 4 to 10 cycles of chemotherapy depending on the response to treatment. The EUA was done after an average of 2 cycles of chemotherapy. In addition to systemic chemotherapy, patients were also given local therapy. This consisted of laser therapy; cryotherapy; intravitreal chemotherapy (melphalan at dose 20-30 mg and methotrexate [MTX] $400 \mathrm{mg}^{[8]}$ and periocular chemotherapy (topotecan 0.5-2 mg). ${ }^{[9]}$ Disease response was assessed at the end of the therapy with MRI study and EUA (patients who had vitreous seeding/local minimal disease were started on local therapy). Patients with relapsed disease were treated on second-line chemotherapy - ifosfamide, vincristine and doxorubicin (IVAD) (vincristine $1.5 \mathrm{mg} / \mathrm{m}^{2}$, doxorubicin $25 \mathrm{mg} / \mathrm{m}^{2}$ and ifosfamide $3000 \mathrm{mg} / \mathrm{m}^{2}$ ). ${ }^{[10]}$ Patients treated will be followed until the age of 10 years.

Statistical analysis was performed using SPSS software (Version 22.0; SPSS, Chicago, IL). Descriptive statistics were reported as frequencies and percentages for quantitative data. Fischer's exact test as used to determine the statistical association between the extent of disease and outcome of the therapy. $P<0.05$ was statistically significant. Diseasefree event was calculated from the end of treatment until the first event of disease progression or relapse.

\section{Results}

A total of 15 patients met the inclusion criteria. The median age of presentation was 20 months (range $2-40$ months). About $66.7 \%(n=10)$ of the patients were male. The most common presenting symptom was leucocoria. It was present in $66.7 \%$ $(n=10)$ of patients. This was followed by the presence of squint in $20 \%(n=3)$ of patients. Unilateral advance disease was seen in four patients, whereas bilateral disease was present in 11 patients. Group D or E was seen in 12 patients (80\%), whereas the rest of the patients had Group B or C.
The association between the involvement of globe (unilateral or bilateral) and stage of the disease was not statistically significant $(P>0.05)$. These results are summarised in Table 2.

Laser therapy was done in 10 patients (66.7\%) along with systemic chemotherapy. However, due to poor therapeutic response, six patients underwent additional treatment in the form of cryotherapy. Intravitreal melphalan was administered to 3 patients (20\%) secondary to persistent vitreous seeding. Likewise, two patients received intravitreal MTX. Periocular topotecan was given to four patients and intravitreal topotecan was administered to one patient. Systemic melphalan for globe salvage was administered to two patients. There was no statistically significant association present between the severity of disease and the outcome of the treatment modalities $(P>0.05)$.

Relapse of intraocular RB was seen in 12 patients (80\%). Among these, nine underwent enucleation, one was started on second-line chemotherapy IVAD and two died before any intervention could be offered. Two-year disease-free survival (DFS) was calculated to be $20 \%$. None of the patients had distant metastasis or secondary malignancies.

\section{Discussion}

$\mathrm{RB}$ is the most common intraocular malignancy in children that can lead to death within 1-2 years, if left

\section{Table 2: Summary of patient characteristics}

\begin{tabular}{l|l|c}
\hline Clinical parameter & Subtype & $\boldsymbol{n}(\%)$ \\
\hline \multirow{2}{*}{ Age } & $<12$ months & $6(40)$ \\
\cline { 2 - 3 } & $>12$ months & $9(60)$ \\
\hline \multirow{2}{*}{ Gender } & Male & $10(66.7)$ \\
\cline { 2 - 3 } & Female & $5(33.3)$ \\
\hline \multirow{2}{*}{ Disease involvement } & Unilateral & $4(26.7)$ \\
\cline { 2 - 3 } & Bilateral & $11(73.3)$ \\
\hline \multirow{2}{*}{ Clinical presentation } & Leucocoria & $10(66.7)$ \\
\cline { 2 - 3 } & Strabismus & $3(20)$ \\
\cline { 2 - 3 } & Proptosis & $2(13.3)$ \\
\hline \multirow{2}{*}{ Disease stage } & Group B or C & $3(20)$ \\
\cline { 2 - 3 } & Group D or E & $12(80)$ \\
\hline
\end{tabular}


untreated. ${ }^{[11]}$ The aim of this study was to determine the outcome of salvage treatment in advanced RB. A total of 15 patients were included in the present investigation. Two-year DFS was found to be $20 \%$. Among patients that had relapse of RB, $75 \%$ underwent enucleation. No statistically significant association was present between the severity of the disease and the outcome of treatment modalities.

Over the pastyears, intra-arterial chemotherapy (IAC) has been developed as a promising alternative instead of systemic chemotherapy for the management of advanced and refractory RB. The main reason behind the popularity of IAC is the avoidance of enucleation. ${ }^{[12]}$ Majority of patients in the present study fell into either Group D or E as per IRCB. This is likely due to delayed presentation to the health-care facility. Similar observations have been made by other investigators working in developing countries. ${ }^{[13,14]}$

In the present study, three patients received intravitreal (IVit) melphalan in addition to systemic therapy. All these patients had recurrence of disease and underwent enucleation. This is contrary to the findings reported by others. Previously, it has been shown that patients receiving IVit along with systemic chemotherapy have good outcomes in terms of globe salvage. ${ }^{[11]}$

Prior investigations have reported beneficial outcomes with the use of high-dose periocular topotecan. These effects have been consistent even in the presence of vitreous seeding. ${ }^{[15]}$ In the present study, three patients received periocular topotecan along with systemic chemotherapy. However, two of these three patients had recurrence and underwent enucleation. Nonetheless, in one patient, globe was salvaged. This is likely because these two patients had relatively advanced disease (Group D or E stage).

The use of periocular chemotherapy (carboplatin), as monotherapy is not recommended. Nonetheless, when used in combination with other modalities, it has shown to result in significant improvements without any major acute or chronic complications. ${ }^{[16]}$ In the present series, one patient with persistent disease received periocular carboplatin along with systemic chemotherapy. However, this patient died due to relapse of disease.

The outcome of salvage therapy with IVit chemotherapy and/or periocular chemotherapy was only $20 \%$. Based on the findings of the present investigation, we recommend upfront or early enucleation for patients with advanced disease in centres that have limited resources or limited expertise in IAC. One of the major limitations of the present study is that it is a retrospective analysis. It was not possible to control clinical visits, number and type of chemotherapy sessions and local treatments or severity of disease. In addition, due to the nature of the study design, it may result in magnification of the positive or negative responses. Nonetheless, in this study, we used records from all the hospital visits to reduce risk of recall or reporting bias. Future investigations on the effectiveness of early or late enucleation for patients with advanced disease are recommended.

\section{Acknowledgments}

None.

\section{References}

1. Dimaras H, Corson TW, Cobrinik D, White A, Zhao J, Munier FL, et al. Retinoblastoma. Nat Rev Dis Primers 2015; 1:15021.

2. Satyanarayana L, Asthana S, Labani S P. Childhood cancer incidence in India: A review of population-based cancer registries. Indian Pediatr 2014;51:218-20.

3. Rao R, Honavar SG. Retinoblastoma. Indian J Pediatr 2017;84:937-44.

4. Dimaras H, Kimani K, Dimba EA, Gronsdahl P, White A, Chan HS, et al. Retinoblastoma. Lancet 2012;379:1436-46.

5. Chen M, Jiang $H$, Zhang J, Shen G, Jiang Y, Li H, et al. Outcome of intra-arterial chemotherapy for retinoblastoma and its influencing factors: $A$ retrospective study. Acta Ophthalmol 2017;95:613-8.

6. Murphree AL. Intraocular retinoblastoma: The case for a new group classification. Ophthalmol Clin North Am 2005;18:41-53.

7. UKCCSG Retinoblastoma Working Group. Guidelines for the Management of Children with Advanced Retinoblastoma Following Primary Enucleation, Version $1.0 ; 2005$. 
8. Manjandavida FP, Shields CL. The role of intravitreal chemotherapy for retinoblastoma. Indian J Ophthalmol 2015;63:141-5.

9. Chantada GL, Fandino AC, Carcaboso AM, Lagomarsino E, de Davila MT, Guitter MR, et al. A phase I study of periocular topotecan in children with intraocular retinoblastoma. Invest Ophthalmol Vis Sci 2009;50:1492-6.

10. Mruthyunjaya $P$, Kingston JE, Hungerford JL. Treatment outcomes and globe salvage using second line chemotherapy in advanced retinoblastoma. Invest Ophthalmol Vis Sci 2006;47:2291.

11. Abramson DH. Chemosurgery for retinoblastoma: What we know after 5 years. Arch Ophthalmol 2011;129:1492-4.

12. Manjandavida FP, Stathopoulos C, Zhang J, Honavar SG, Shields CL. Intra-arterial chemotherapy in retinoblastoma a paradigm change. Indian J Ophthalmol 2019;67:740-54.

13. Gao J, Zeng J, Guo B, He W, Chen J, Lu F, et al. Clinical presentation and treatment outcome of retinoblastoma in children of South Western China. Medicine (Baltimore) 2016;95:e5204.

14. Waddell KM, Kagame K, Ndamira A, Twinamasiko A, Picton SV, Simmons IG, et al. Clinical features and survival among children with retinoblastoma in Uganda. Br J Ophthalmol 2015;99:387-90.

15. Sthapit PR, Rao R, Honavar SG. Periocular topotecan for vitreous seeds in retinoblastoma. Indian J Ophthalmol 2018;66:1833-8.

16. Marr BP, Dunkel IJ, Linker A, Abramson DH. Periocular carboplatin for retinoblastoma: Longterm report (12 years) on efficacy and toxicity. Br J Ophthalmol 2012;96:881-3.

\section{Authorship Contributions}

Conceived and designed the analysis: PR, SJK; Collected the data: PR; Contributed data or analysis tools: PR, SJK; Performed the analysis: PR; Wrote the paper: PR 